\title{
C-Reactive Protein Mediates the Effect of Serum Progesterone on Obesity for Men and Postmenopausal Women in Henan Rural Cohort Study
}

This article was published in the following Dove Press journal:

Journal of Inflammation Research

\author{
Luting $\mathrm{Nie}^{\mathrm{I}, *}$ \\ Dandan $\mathrm{Wei}^{2, *}$ \\ Pengling Liu ${ }^{2}$ \\ Li Zhang ${ }^{2}$ \\ Keliang Fan ${ }^{2}$ \\ Yu Song' \\ Mian Wang' \\ Lulu Wang ${ }^{2}$ \\ Qingqing $X u^{2}$ \\ Juan Wang ${ }^{2}$ \\ Xiaotian $\mathrm{Liu}^{2}$ \\ Linlin $\mathrm{Li}^{2}$ \\ Zhenxing $\mathrm{Mao}^{2}$ \\ Hui Huang' \\ Chongjian Wang (iD ${ }^{2}$ \\ Wenqian Huo' \\ 'Department of Occupational and \\ Environmental Health, College of Public \\ Health, Zhengzhou University, \\ Zhengzhou, Henan, People's Republic of \\ China; ${ }^{2}$ Department of Epidemiology and \\ Biostatistics, College of Public Health, \\ Zhengzhou University, Zhengzhou, \\ Henan, People's Republic of China \\ *These authors contributed equally to \\ this work
}

Correspondence: Wenqian Huo Department of Occupational and Environmental Health Sciences, 100

Kexue Avenue, Zhengzhou, Henan,

45000I, People's Republic of China

Tel +86 37I 6778I452

Fax +86 37I 6778I868

Email huowenqian@zzu.edu.cn
Purpose: Studies of progesterone with obesity have been lacking, and no prior studies have investigated progesterone and C-reactive protein in rural natural populations. This study aimed to investigate the association of serum progesterone with obesity based on anthropometric parameters in Henan Rural Cohort, then further to explore the potential role of C-reactive protein in this association.

Patients and Methods: A total of 4687 participants (2474 men and 2213 postmenopausal women) from the Henan Rural Cohort study were included. Logistic regression analysis, linear regression analysis, and restricted cubic splines were performed to estimate the relationships between progesterone, C-reactive protein, obesity, and obesity-related parameters. Mediation analysis was carried out to assess the intermediary role of C-reactive protein played in the association between progesterone and obesity.

Results: After controlling for confounders, the odds ratios of obesity no matter how obesity was defined was associated with progesterone $(P$ trend $<0.05)$. Moreover, progesterone was related to obesity-related parameters. Also, C-reactive protein increased with the elevation of progesterone, and C-reactive protein was associated with obesity no matter how obesity was defined $(P$ trend $<0.001$ ). Mediation analysis indicated that hsCRP had the mediating effects on the obesity, and the proportion of the effects were $15.35 \%$ and full mediating effect (defined by body mass index), $15.46 \%$ and $12.24 \%$ (defined by waist circumference), $19.14 \%$ and $12.12 \%$ (defined by waist-to-hip ratio), $23.81 \%$ and $15.09 \%$ (defined by waistto-height ratio) for men and postmenopausal women, relatively.

Conclusion: In the Chinese rural men and postmenopausal women population, progesterone and C-reactive protein are related to obesity. We firstly found C-reactive protein partly mediates the effect of progesterone on obesity, indicating that systemic inflammation played a critical role in the association.

Keywords: sex hormone, obesity, C-reactive protein, mediation effect

\section{Introduction}

Obesity is a disorder of energy metabolism, which is associated with rising mortality and morbidity of numerous chronic non-communicable diseases, has become a serious public health problem. ${ }^{1-3}$ Since 1975 , obesity has increased at an alarming rate and nearly tripled globally. ${ }^{4}$ In China, especially in rural, the resource-limited settings, the same prevalence trend of obesity also exists. ${ }^{5}$ It is reported that overweight and obesity are reaching nearly $89 \%$ of men and $85 \%$ of women 
respectively by $2030{ }^{6}$ There are several anthropometric indices to define obesity, such as body mass index (BMI), waist circumference (WC), waist-to-hip ratio (WHR), and waist-to-height ratio (WHtR). ${ }^{7-10}$ Beyond this, the rates of obesity varied by obese indicators, and different obesity indicators have different meanings. ${ }^{11-13}$ So, it is worth using different kinds of obese indicators when studying obesity.

Some epidemiologic studies, as well as several experimental evidence, suggest that endocrine hormones may be correlate with obesity development. Both vitro and vivo studies have shown that as a major sex hormone, progesterone actively participates in various aspects of metabolism within adipose tissue, such as metabolism, distribution, and accumulation of adipose tissue. ${ }^{14,15}$ Some studies have found that progesterone could bind to the progesterone receptor in adipose tissue, causing lipid storage and adipose tissue accumulation by directly regulating lipoprotein lipase. ${ }^{14-16}$ However, the results from epidemiologic studies have been discrepant. For example, Esfahlan and Clark found that serum progesterone and the use of depot-medroxyprogesterone acetate were positively related to obesity, ${ }^{17,18}$ while Blanchette and Goh reported that progesterone was negatively linked to obesity in men and pregnant women. ${ }^{19,20}$ Moreover, the research on the relationship between natural progesterone and obesity in the Chinese rural population is limited, therefore, further studies are warranted.

Chronic low-grade inflammation, involved in many pathological processes of obesity and insulin resistance, will increase the risk of these diseases. C-reactive protein (CRP), an essential key in the innate immune response, is the sensitive and most accessible biomarker to identify individuals with systemic inflammation. ${ }^{21}$ Recently, two prospective cohort studies have found that luteal progesterone in premenopausal women or using progestin combined with estrogen in postmenopausal women was related to a high level of CRP. ${ }^{22,23}$ However, it is unclear whether the natural progesterone is correlated with the increased CRP in the total population including not only women but also men. Also, published studies reported that CRP was positively associated with increased risk of obesity, and cardiovascular events, such as myocardial infarction and stroke. ${ }^{24}$ Studies have observed that compared with the normal population, in those people with obesity and related diseases, the concentration of inflammatory markers such as CRP is higher. ${ }^{25,26}$ Yet few studies have explored the role of CRP played in the relationship between progesterone and obesity. The role serum CRP is not yet as clear.

Taken together, the current direct evidence on the association between progesterone and obesity is still limited, and the mechanisms underlying this relationship remain largely unclear and are likely complex. Therefore, we undertook a cross-sectional study to explore the doseresponse association between progesterone and obesity among 4687 adult rural residents in China. Besides, we further explored the possible mediating effect of systemic inflammation on the relationship.

\section{Patients and Methods Study Design and Study Population}

The study subjects are from the Henan Rural Cohort, an ongoing, population-based cohort study, which is designed and conducted to analyze the potential risk factors of chronic non-communicable diseases among rural China. Detail information has been described elsewhere. ${ }^{27} \mathrm{We}$ recruited 6670 adult residents who participated in the Henan cohort survey in Suiping county in 2016. After excluding missing data on serum progesterone $(n=410)$, premenopausal women (as progestogen levels vary hugely with the menstrual cycle) $(\mathrm{n}=1336)$ and missing information on menopause data $(\mathrm{n}=19)$, taking oral contraceptive steroids or hormone replacement therapy $(\mathrm{n}=28)$, smoking women $(\mathrm{n}=8)$, participants with serum hsCRP $>10 \mathrm{mg} / \mathrm{L}$ $\left(\mathrm{n}=148\right.$ (as such high levels are likely to be abnormal)), ${ }^{28}$ and with missing data on BMI, WC, and vegetable and fruit intake $(\mathrm{n}=41)$, we finally enrolled 4687 participants (2474 men and 2213 postmenopausal women). The Zhengzhou University Life Science Ethics Committee approved this research protocol. (Ethics approval code: [2015] MEC (S128)).

\section{Data Collection}

All participants read an information sheet and completed and signed a consent form prior to accessing the questionnaire. The questionnaire included information on sociodemographic characteristics, behavioral and lifestyle factors, diet, medical history, and personal as well as family history of the diseases. The sociodemographic characteristics included age, sex, education level (below than primary school, primary school, and junior secondary or above), marital status (married/cohabitation and widowed/single/ divorced/separation), and per capita monthly income $(<500$ RMB, 500-999 RMB, $\geq 1000$ RMB). Behavioral and 
lifestyle factors included smoking habit (current, never, or former), drinking habit (current, never or former), and physical activity (low, mediate, high). Dietary habits included adequate vegetable and fruit intake (high ( $\geq 500 \mathrm{~g} /$ day) and low $(<500 \mathrm{~g} /$ day) as well as high-fat diet (yes $(\geq 75 \mathrm{~g} /$ day) and no $(<75 \mathrm{~g} /$ day $)){ }^{29}$ Women were asked to give information about their menopausal status.

The details about anthropometric measurements have been reported in elsewhere. ${ }^{27}$ In brief, anthropometric measurements, including height, weight, and $\mathrm{WC}$ were obtained, according to a standard protocol. $^{30}$ Anthropometry (height, weight, WC, and hip circumference) was measured by keeping participants without shoes and only in light clothing. WC and hip circumference were measured $1 \mathrm{~cm}$ above the navel and around the maximum circumference of the buttocks. The measurement for each indicator was repeated twice, and the average value was taken for statistical analysis.

\section{Laboratory Measurements}

Blood samples were drawn after more than $8 \mathrm{~h}$ of overnight fasting. Next, part of the blood samples was centrifuged for 10 minutes at relative centrifugal force $2000 \mathrm{~g}$ and room temperature, and then serum samples were forwarded clinical laboratory center to measure. The levels of serum hsCRP were detected by immunoturbidimetry (Cobas c501, Roche, Switzerland). Following quartiles of hsCRP levels, hsCRP concentrations were divided into four groups with the lowest quartile group as the reference group. And the other part of the blood samples was centrifuged for 10 minutes at $3000 \mathrm{rpm}$ and $4^{\circ} \mathrm{C}$, and then serum samples were stored at $-80^{\circ} \mathrm{C}$. Progesterone was measured with a liquid chromatography-tandem mass spectrometry (LC-MS/MS) method (Waters e2695, Waters XEVO TQ-S system, Waters). ${ }^{31}$ The limit of detection (LOD) was $0.02 \mathrm{ng} / \mathrm{mL}$ for progesterone. The detected concentration below the LOD or undetected was replaced by $1 / 2 \mathrm{LOD}$ as $0.01 \mathrm{ng} / \mathrm{mL}$. Following the distribution of progesterone levels, this study divided all participants into four groups based on the quartile of progesterone levels in men and postmenopausal women, and the first quartile was the reference group.

\section{Outcomes}

BMI was derived from weight and square of height. WHR was derived from WC and hip circumference, and WHtR was derived from WC and height. A BMI $\geq 28.0 \mathrm{~kg} / \mathrm{m}^{2}$, a WC $\geq 90 \mathrm{~cm}$ in men or $\geq 80 \mathrm{~cm}$ in women, a WHR $\geq 90 \%$ in men or $\geq 85 \%$ in women, and a WHtR $\geq 50 \%$ was defined as obesity. ${ }^{7-10}$

\section{Statistical Analysis}

Continuous variables were presented as the mean \pm standard deviation (SD), and the categorical variables were presented as frequency and percentage. Group differences in continuously scaled measures and categorical variables were assessed by independent-samples t-tests and chi-square tests, respectively. Serum progesterone and hsCPR have been subjected to natural logarithmic transformation in the subsequent analysis due to their skewed distributions.

We used logistic regression analysis to compute the associations of serum progesterone and hsCRP levels with the odds ratios of obesity. Tests for trend were performed by entering the categorical variables as continuous variables in logistic regression models to assess the relationship across increasing quartiles. Linear regression was used to further assess the correlation of progesterone levels with obesityrelated parameters (BMI, WC, WHR, and WHtR) and inflammatory markers (hsCRP). The nonlinearity of the effect of continuous progesterone and hsCRP and obesity were evaluated with the restricted cubic spline with 3 knots at fixed percentiles $(25 \%, 50 \%$, and $75 \%) ; 0.45 \mathrm{ng} / \mathrm{mL}$ and $0.67 \mathrm{mg} / \mathrm{L}$ were set as the reference values for progesterone and hsCRP based on distributions. The model was adjusted for age, sex, marital status, education level, average monthly income, smoking habit, drinking habit, high vegetable and fruits intake, high fat diet, and physical activity.

To determine whether the relationship of serum progesterone on obesity was mediated by hsCRP, we conducted mediation analysis, which was conducted running the PROCESS with 5000 bootstraps resamples as previously depicted in elsewhere ${ }^{32}$ by adjusting the same confounders used in the above-mentioned adjusted model.

Other studies have reported lower amounts of progesterone in men and a decrease in progesterone with advancing age for men. ${ }^{33}$ To address the potential modifiers of age, sensitivity analyses were conducted to investigate the relationship between progesterone and obesity and obesity-related parameters, the relationship between hsCRP and obesity and obesity-related parameters, and the mediating effect of hsCRP in the association between progesterone and obesity among different age groups $(<46$ years, 46-65 years, and $>65$ years). All data analysis was conducted in R software version 4.0.0 and SPSS software, version 21.0. And all statistical significances were set a $P$ value $<0.05$ at two tail. 


\section{Results}

\section{Characteristics of Study Population}

Characteristics of the study participants are presented in Table 1. The mean (SD) age of men and postmenopausal women was $57.52(10.85)$ and $61.12(7.95)$ years old. Men had a higher level of WC, WHR and WHtR while postmenopausal women had a higher level of hsCRP, progesterone, and BMI. Compared with postmenopausal women, men tended to have higher education level, higher per capita monthly income, higher proportion of current smokers, drinkers, high fat diet, and physical activity. No significant differences in the other selected variables.

Table I Characteristics of Study Population by Gender

\begin{tabular}{|c|c|c|c|}
\hline \multirow[t]{2}{*}{ Variable } & \multirow{2}{*}{$\begin{array}{l}\text { Men } \\
(N=2747)\end{array}$} & \multirow{2}{*}{$\begin{array}{l}\text { Postmenopausal Women } \\
(N=2213)\end{array}$} & \multirow[t]{2}{*}{$\boldsymbol{P}$} \\
\hline & & & \\
\hline Age (years), mean $\pm S D$ & $57.52 \pm 10.85$ & $61.12 \pm 7.95$ & $<0.001^{\mathrm{a}}$ \\
\hline Educational attainment, n (\%) & & & $<0.001^{\mathrm{b}}$ \\
\hline Never attended school & $279(11.28)$ & $658(29.73)$ & \\
\hline Primary school & $620(25.06)$ & $697(31.50)$ & \\
\hline Junior secondary and above & $1575(63.66)$ & $858(38.77)$ & \\
\hline Marital status, n (\%) & & & $0.122^{b}$ \\
\hline Married/cohabiting & $2211(89.37)$ & 1946 (87.93) & \\
\hline Widowed/single/divorced/separation & $263(10.63)$ & $267(12.07)$ & \\
\hline Per capita monthly income, n (\%) & & & $0.021^{b}$ \\
\hline$<500, \mathrm{RMB}$ & $807(32.62)$ & $794(35.88)$ & \\
\hline $500 \sim, \mathrm{RMB}$ & $697(28.17)$ & $63 I(28.5 I)$ & \\
\hline 1000 , RMB & $970(39.21)$ & $788(35.61)$ & \\
\hline Current smoker, n (\%) & I I 74 (47.45) & $0(0.00)$ & $<0.001^{\mathrm{b}}$ \\
\hline Current drinker, n (\%) & $843(34.07)$ & $35(1.58)$ & $<0.001^{\mathrm{b}}$ \\
\hline High fat diet, n (\%) & $782(31.6 I)$ & $431(19.48)$ & $<0.001^{\mathrm{b}}$ \\
\hline Adequate vegetable and fruit intake, $n(\%)$ & $1647(66.57)$ & 1452 (65.6I) & $0.488^{b}$ \\
\hline Physical activity, n (\%) & & & $<0.001^{\mathrm{b}}$ \\
\hline Low & $656(26.52)$ & $394(17.80)$ & \\
\hline Mediate & $927(37.47)$ & 1322 (59.74) & \\
\hline High & $891(36.01)$ & $497(22.46)$ & \\
\hline $\mathrm{hsCRP}(\mathrm{mg} / \mathrm{L})$, mean $\pm \mathrm{SD}$ & $1.62 \pm 1.58$ & $1.73 \pm 1.61$ & $<0.001^{\mathrm{a}}$ \\
\hline Progesterone $(\mathrm{ng} / \mathrm{mL})$, mean $\pm \mathrm{SD}$ & $0.90 \pm 0.97$ & $1.02 \pm 1.43$ & $0.001^{\mathrm{a}}$ \\
\hline $\mathrm{BMI}(\mathrm{kg} / \mathrm{m} 2)$, mean $\pm \mathrm{SD}$ & $23.58 \pm 3.19$ & $23.87 \pm 3.34$ & $0.003^{\mathrm{a}}$ \\
\hline$W C(\mathrm{~cm})$, mean $\pm S D$ & $83.85 \pm 9.88$ & $82.13 \pm 9.78$ & $0.00 I^{\mathrm{a}}$ \\
\hline WHR (\%), mean \pm SD & $89.07 \pm 6.47$ & $88.20 \pm 6.76$ & $0.00 I^{\mathrm{a}}$ \\
\hline $\mathrm{WH} t \mathrm{tR}(\%)$, mean $\pm \mathrm{SD}$ & $50.37 \pm 5.71$ & $53.15 \pm 6.20$ & $0.001^{\mathrm{a}}$ \\
\hline
\end{tabular}

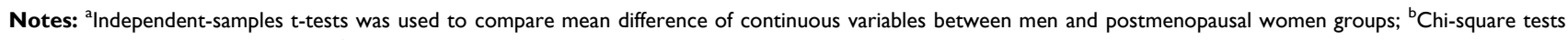
was used to test the distributions of categorical variables between men and postmenopausal women groups.

Abbreviations: BMI, body mass index; WC, waist circumference; WHR, waist-to-hip ratio; WHtR, waist-to-height ratio. 


\section{Associations of Serum Progesterone with Obesity and Obesity-Related Parameters}

The changes and $95 \%$ CI of obesity and four obesity parameters with per IQR increment of ln-progesterone are shown in Figures 1 and 2. The results presented positive associations between progesterone and obesity no matter how obesity was defined and four obesity-related parameters. Such as, in the adjusted model, when obesity was defined by BMI, lnprogesterone was significantly associated with obesity and the OR $(95 \% \mathrm{CI})$ was $1.24(1.02,1.51)$ and $1.17(0.99,1.38)$ in men and postmenopausal women. And a one-unit increase in ln-progesterone was associated with a $0.26 \mathrm{~kg} / \mathrm{m}^{2}(95 \%$ CI: $0.11,0.42)$ and $0.33 \mathrm{~kg} / \mathrm{m}^{2}$ (95\% CI: $\left.0.18,0.49\right)$ increase in BMI in men and postmenopausal women; and compared with the first quartile, the fourth quartile of $\ln$-progesterone was related to a $0.45 \mathrm{~kg} / \mathrm{m}^{2}$ (95\% CI: $\left.0.11,0.79\right)$ and $0.83 \mathrm{~kg} / \mathrm{m}^{2}$ (95\% CI: $\left.0.43,1.22\right)$ increase in BMI in men and postmenopausal women.
As shown in Supplementary Figure 1, for postmenopausal women, ORs of obesity defined by WC, WHR and WHtR increased gradually with an incremental in the serum concentration of progesterone in all participants (all $P$ for overall association $<0.05$, and all $P$ for nonlinearity $<0.05$ ), indicating a significant nonlinear dose-response relationship while no significant dose-response relationship was found between progesterone and obesity defined by BMI $(P$ for overall association $>0.05$ ). For men, liner dose-response relationships were found between progesterone with obesity defined all obesity-related parameters (all $P$ for overall association $<0.05$, and all $P$ for nonlinearity $>0.05$ ).

\section{Associations of Serum Progesterone with hsCRP}

The results of the linear regression of the relationship between In-progesterone and hsCRP are displayed in Table 2. After adjusting all confounding factors, each

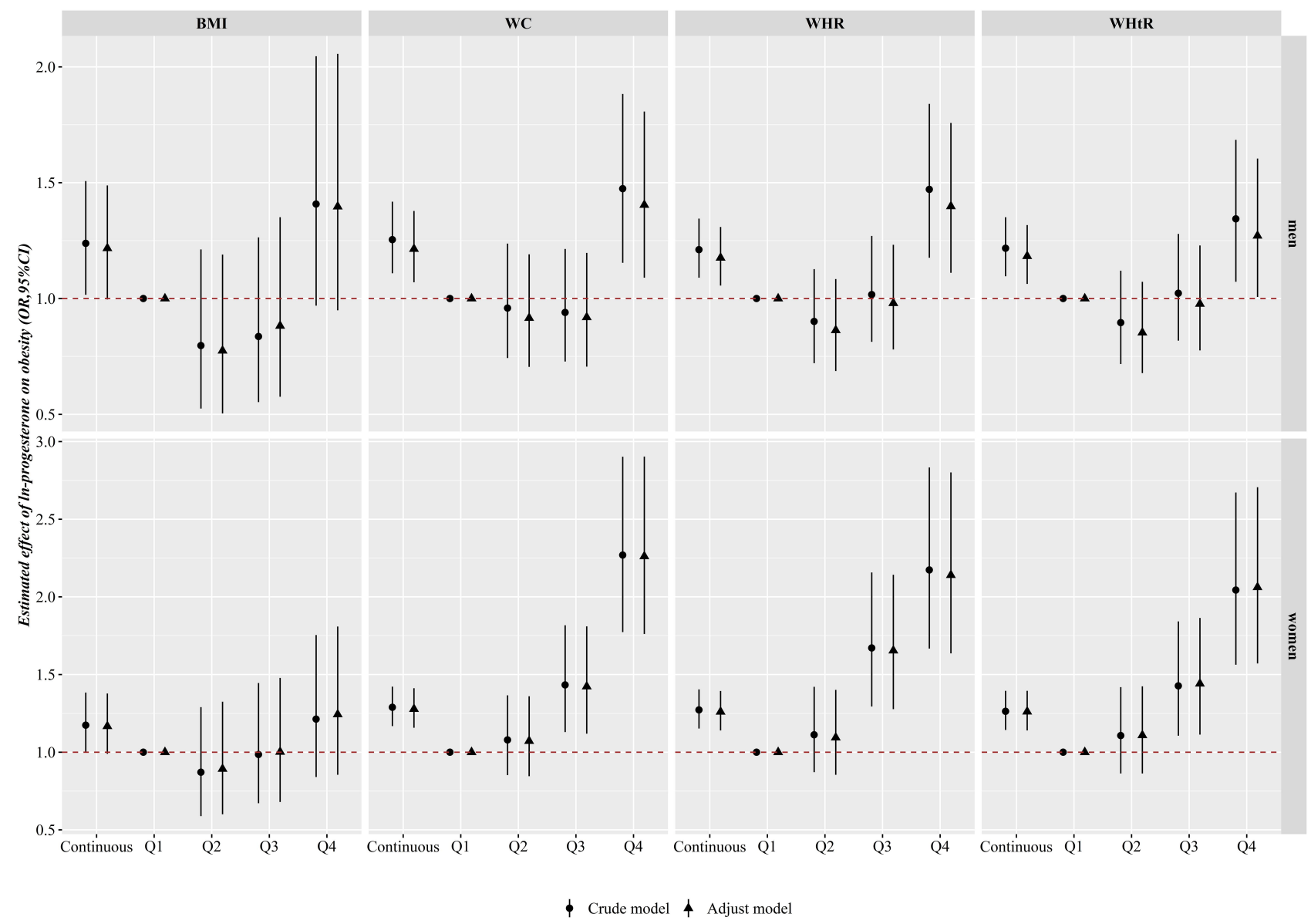

Figure I Odds ratios of obesity according to the quartiles of In-progesterone by gender. Adjusted for age, marital status, education level, average monthly income, smoking habit, drinking habit, high vegetables and fruits intake, high fat diet, physical activity.

Abbreviations: BMI, body mass index; WC, waist circumference; WHR, waist-to-hip ratio; WHtR, waist-to-height ratio; QI, the first quartile; Q2, the secondly quartile; Q3, the thirdly quartile; Q4, the fourthly quartile. 


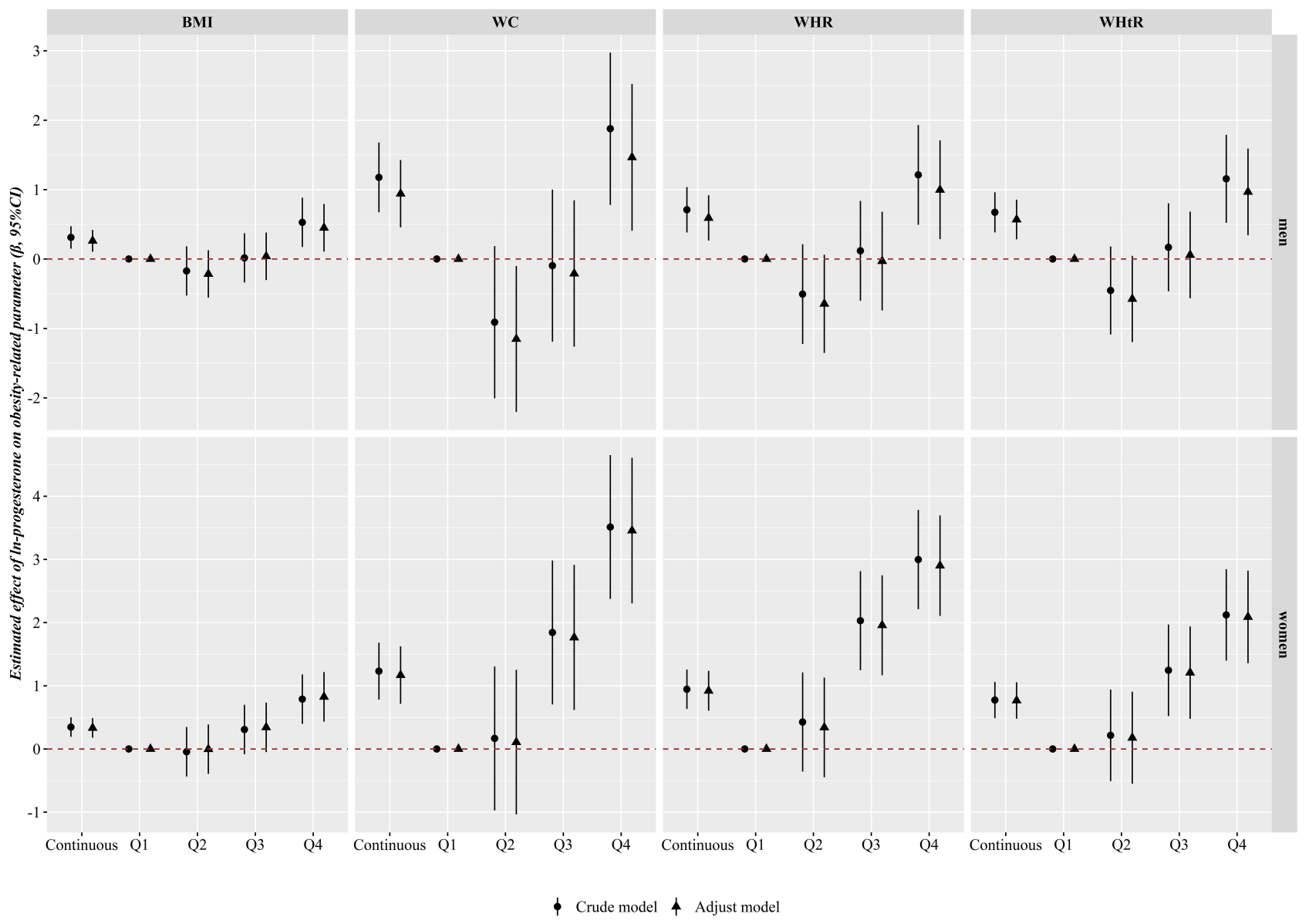

Figure 2 Partial regression confident of obesity-related parameters according to the quartiles of In-progesterone by gender. Adjusted for age, marital status, education level, average monthly income, smoking habit, drinking habit, high vegetables and fruits intake, high fat diet, physical activity.

Abbreviations: BMI, body mass index; WC, waist circumference; WHR, waist-to-hip ratio; WHtR, waist-to-height ratio; QI, the first quartile; Q2, the secondly quartile; Q3, the thirdly quartile; Q4, the fourthly quartile.

1-unit increase in ln-progesterone was positively related to a $14 \%$ and $10 \%$ increased hsCRP levels in in men and postmenopausal women, respectively. Compared with the first quartile of serum progesterone, the estimated changes (95\% CI) of hsCRP in the fourth quartile (Q4) of hsCRP were $0.34(0.16,0.51)$ and $0.26(0.07,0.45)$ in men and postmenopausal women, respectively.

\section{Associations of hsCRP Levels with Obesity}

Table 3 presents the adjusted odds ratios of obesity with ln-hsCRP quartiles. The results displayed that $1 n$-hsCRP was associated with four kinds of obesity. For instance, in the adjusted model, ln-hsCRP was significantly correlated with obesity defined by BMI (men: OR $(95 \% \mathrm{CI})$ : Q2 vs Q1: 2.10 (1.25, 3.52); Q3 vs Q1: 3.30 (2.00, 5.42); Q4 vs Q1: 5.03 (3.11, 8.13); postmenopausal women: OR (95\% CI): Q2 vs Q1: 2.22 (1.23, 3.99); Q3 vs Q1: 3.80 (2.19,
6.60); Q4 vs Q1: $7.77(4.59,13.17))$. Also, nonlinear doseresponse relationships were found between hsCRP and obesity defined by all obesity-related parameters in the adjusted model (all $P$ for overall association $<0.05$, and all $P$ for nonlinearity $<0.05$ ), except obesity defined by BMI for postmenopausal women (all $P$ for overall association $>0.05$ ) (Supplementary Figure 2).

\section{Role of hsCRP in the Association Between Progesterone and Obesity}

In the above-presented analysis, we found that the level of progesterone was significantly associated with hsCRP and obesity. We further performed a mediation analysis to evaluate the possible mediation effects of hsCRP on associations of progesterone with obesity. Table 4 presents the mediation effect of hsCRP after adjustment, indicating that hsCRP significantly mediated the associations of progesterone and obesity in men and postmenopausal women. 
Table 2 Associations of Serum Ln-Progesterone Levels with hsCRP by Gender

\begin{tabular}{|c|c|c|c|}
\hline \multirow[t]{2}{*}{ Outcomes } & \multirow[t]{2}{*}{ Subjects } & \multicolumn{2}{|c|}{ Linear Regression $\beta$ Coefficients } \\
\hline & & Crude Model & $\begin{array}{l}\text { Adjusted } \\
\text { Model }\end{array}$ \\
\hline \multicolumn{4}{|l|}{ Men } \\
\hline Continuous & 2474 & $0.14(0.06,0.22)^{*}$ & $0.14(0.06,0.22)^{*}$ \\
\hline QI & 618 & reference & reference \\
\hline Q2 & 619 & $0.13(-0.04,0.31)$ & $0.13(-0.05,0.30)$ \\
\hline Q3 & 618 & $0.21(0.03,0.38)^{*}$ & $0.21(0.03,0.38)^{*}$ \\
\hline Q4 & 619 & $0.33(0.16,0.5 \mathrm{I})^{*}$ & $0.34(0.16,0.5 \mathrm{I})^{*}$ \\
\hline \multicolumn{4}{|c|}{$\begin{array}{l}\text { Postmenopausal } \\
\text { women }\end{array}$} \\
\hline Continuous & 2213 & $0.10(0.02,0.17)^{*}$ & $0.10(0.02,0.17)^{*}$ \\
\hline QI & 554 & reference & reference \\
\hline Q2 & 553 & $0.04(-0.15,0.23)$ & $0.03(-0.16,0.22)$ \\
\hline Q3 & 553 & $0.10(-0.09,0.29)$ & $0.09(-0.11,0.28)$ \\
\hline Q4 & 553 & $0.27(0.08,0.46)^{*}$ & $0.26(0.07,0.45)^{*}$ \\
\hline
\end{tabular}

Notes: Crude model, OR, 95\% Cl: no adjust. Adjusted model, OR, 95\% Cl: adjusted for age, marital status, education level, average monthly income, smoking habit, drinking habit, high vegetables and fruits intake, high fat diet, physical activity. $* P<0.05$.

For men, the proportions of the mediation effect of hsCRP for obesity defined by BMI, WC, WHR, and WHtR was $15.35 \%, 15.46 \%, 19.14 \%$, and $23.81 \%$, respectively. For the postmenopausal women, the proportions of the mediation effect of hsCRP for obesity defined by WC, WHR, and WHtR was $12.24 \%, 12.12 \%$, and $15.09 \%$, and for obesity defined by BMI, hsCRP had a full mediating effect.

\section{Sensitivity Analysis}

A detailed description of the results of sensitivity analyses can be found in Supplementary materials.

\section{Discussion}

As far as we are aware, this study is the first one to explore the mediation effect of hsCRP on the relationship between serum progesterone and obesity with four obesity-related parameters in rural Chinese adults. The present study demonstrated a positive relationship between progesterone and obesity regardless of how obesity was defined. Besides, serum progesterone was positively related to serum hsCRP, and serum hsCRP was also positively associated with obesity. The results from the mediation analysis demonstrated that serum hsCRP was an important mediator of the association between serum progesterone and obesity, indicating that systemic inflammation played a critical role in the association of progesterone with obesity. In case of agestratified analyses in men, the results suggested that only in men aged 45-65 years, progesterone and hsCRP significantly increased the risk of obesity, and the relationship between progesterone and obesity was mediated by hsCRP.

For now, we are the first study to simultaneously examine progesterone levels and obesity defined by BMI, WC, WHR, and WHtR. One large prospective study assessing the long-term effect of depot medroxyprogesterone acetate (DMPA) contraception showed that using the medicine was related to greater weight gain. ${ }^{18}$ Also, in one case-control study including 70 women who had breast cancer, the serum level of progesterone in obese cases was found higher than non-obese controls. ${ }^{17}$ These results are consistent with our findings. However, in a maternity hospital-based study, Goh et al reported an inverse relationship of serum progesterone level with maternal obesity defined by BMI in early pregnancy. ${ }^{20}$ Another previous study conducted by Blanchette et al showed that circulating progesterone was negatively linked to indicators of obesity such as WC and BMI among 38 men aged 44.5 \pm 9.9 years old. ${ }^{19}$ The discrepant results between ours, Blanchette's, and Goh's may be attributable to the different investigating populations and different sample sizes. Goh only focused on pregnant women, as well as the Blanchette's no more than 100 middle-aged men, but our study consisted of 4687 men and postmenopausal women. In this large population-based study, we recognized that besides obesity defined by BMI, increasing serum progesterone was also correlate with a higher risk of obesity defined by central adiposity parameters (WC, WHR, and WHtR), but little is known about the specific mechanisms. Obviously, further investigations are necessary to determine the mechanism. The present study fills the research gap on the evidence of the research between natural progesterone and obesity in natural populations. Published literatures have proved that progesterone actively participated in lipid metabolism. The storage, and accumulation of adipose tissue might be caused by the progesterone receptor in adipose tissues, which can bound to progesterone, and then regulate lipoprotein lipase. Besides, cAMP cascade, and phosphoinositide cascade are also critical for 
Table 3 Associations of Serum Ln-hsCRP Levels with Obesity Defined by BMI, WC, WHR, and WHtR by Gender

\begin{tabular}{|c|c|c|c|c|c|c|}
\hline \multirow[t]{3}{*}{ Outcomes } & \multicolumn{6}{|c|}{ Logistic Regression ORs (95\% Cl) } \\
\hline & \multirow[t]{2}{*}{ Subjects } & \multicolumn{2}{|l|}{ Men } & \multirow[t]{2}{*}{ Subjects } & \multicolumn{2}{|c|}{ Postmenopausal Women } \\
\hline & & Crude Model & Adjusted Model & & Crude Model & Adjusted Model \\
\hline \multicolumn{7}{|l|}{ BMI } \\
\hline Continuous & 2474 & $\mathrm{I} .87(\mathrm{I} .57,2.22) *$ & $1.99(1.66,2.38) *$ & 2213 & $2.30(1.93,2.74) *$ & $2.35(1.97,2.80) *$ \\
\hline QI & 676 & reference & reference & 507 & reference & reference \\
\hline Q2 & 618 & $2.08(1.25,3.47) *$ & $2.10(1.25,3.52) *$ & 549 & $2.14(1.19,3.85) *$ & $2.22(1.23,3.99) *$ \\
\hline Q3 & 592 & $3.12(1.92,5.07) *$ & $3.30(2.00,5.42) *$ & 577 & $3.60(2.08,6.23) *$ & $3.80(2.19,6.60) *$ \\
\hline Q4 & 588 & $4.47(2.79,7.14) *$ & $5.03(3.11,8.13) *$ & 580 & $7.44(4.4 I, 12.56) *$ & $7.77(4.59,13.17) *$ \\
\hline$P$-trend & & $<0.001$ & $<0.001$ & & $<0.001$ & $<0.001$ \\
\hline \multicolumn{7}{|l|}{ WC } \\
\hline Continuous & 2474 & $1.73(1.54,1.94) *$ & $1.83(1.62,2.06) *$ & 2213 & $2.29(2.02,2.60) *$ & $2.31(2.04,2.63) *$ \\
\hline QI & 676 & reference & reference & 507 & reference & reference \\
\hline Q2 & 618 & $1.85(1.40,2.43) *$ & $1.83(1.38,2.42) *$ & 549 & $2.22(1.73,2.84) *$ & $2.25(1.75,2.89) *$ \\
\hline Q3 & 592 & $2.58(1.97,3.38) *$ & $2.66(2.0 \mathrm{I}, 3.5 \mathrm{I}) *$ & 577 & $3.74(2.9 \mathrm{I}, 4.8 \mathrm{I}) *$ & $3.85(2.98,4.96) *$ \\
\hline Q4 & 588 & $3.33(2.56,4.35) *$ & $3.66(2.78,4.82) *$ & 580 & $5.54(4.26,7.20) *$ & $5.67(4.35,7.38) *$ \\
\hline$P$-trend & & $<0.001$ & $<0.001$ & & $<0.001$ & $<0.001$ \\
\hline \multicolumn{7}{|l|}{ WHR } \\
\hline Continuous & 2474 & $1.79(1.60,1.99) *$ & $1.83(1.64,2.05) *$ & 2213 & $2.13(1.86,2.44) *$ & $2.15(1.87,2.46) *$ \\
\hline QI & 676 & reference & reference & 507 & reference & reference \\
\hline Q2 & 618 & $2.58(2.05,3.24) *$ & $1.66(1.32,2.09) *$ & 549 & $1.95(1.52,2.50) *$ & $1.98(1.54,2.54) *$ \\
\hline Q3 & 592 & $3.23(2.57,4.07) *$ & $2.58(2.05,3.26) *$ & 577 & $3.17(2.44,4.11) *$ & $3.20(2.47,4.16) *$ \\
\hline Q4 & 588 & $0.00(0.00,0.00) *$ & $3.38(2.66,4.28) *$ & 580 & $4.33(3.29,5.70) *$ & $4.39(3.33,5.79) *$ \\
\hline$P$-trend & & $<0.001$ & $<0.001$ & & $<0.001$ & $<0.001$ \\
\hline \multicolumn{7}{|l|}{ WHtR } \\
\hline Continuous & 2474 & $1.95(1.74,2.18) *$ & $2.02(1.80,2.27) *$ & 2213 & $2.40(2.09,2.76) *$ & $2.43(2.11,2.80) *$ \\
\hline Q। & 676 & reference & reference & 507 & reference & reference \\
\hline Q2 & 618 & $\mathrm{I} .83(\mathrm{I} .47,2.29) *$ & $\mathrm{I} .80(\mathrm{I} .44,2.26) *$ & 549 & $2.53(1.96,3.25) *$ & $2.60(2.02,3.36) *$ \\
\hline Q3 & 592 & $2.65(2.11,3.33) *$ & $2.69(2.13,3.39) *$ & 577 & $3.59(2.77,4.66) *$ & $3.68(2.83,4.78) *$ \\
\hline Q4 & 588 & $3.69(2.92,4.67) *$ & $3.94(3.09,5.02) *$ & 580 & $6.24(4.68,8.32) *$ & $6.42(4.81,8.59) *$ \\
\hline$P$-trend & & $<0.001$ & $<0.001$ & & $<0.001$ & $<0.001$ \\
\hline
\end{tabular}

Notes: Crude model, OR, $95 \% \mathrm{Cl}$ : no adjust. Adjusted model, OR, $95 \% \mathrm{Cl}$ : adjusted for age, marital status, education level, average monthly income, smoking habit, drinking habit, high vegetables and fruits intake, high fat diet, physical activity. $* P<0.05$.

the regulation of lipid. Progesterone can work with estrogen to reduce fat breakdown and promote lipid production by affecting both cascades. ${ }^{14-16}$
CRP has been considered as a representative biomarker of inflammatory that reflects potential biological mechanism. ${ }^{34}$ The potential relationships between high CRP levels and 
Table 4 Mediation Analysis of the Relationship Between Serum Ln-Progesterone Levels and Obesity by hsCRP by Gender

\begin{tabular}{|c|c|c|c|c|c|}
\hline Parameters & Total Effect ${ }^{a}$ & Direct Effect ${ }^{\text {a }}$ & Indirect Effect ${ }^{\text {a }}$ & PE* & $P^{a}$ \\
\hline \multicolumn{6}{|l|}{ Men } \\
\hline BMI & $0.202(0.00 \mathrm{I}, 0.404) *$ & $0.158(-0.043,0.360)$ & $0.031(0.013,0.053) *$ & $15.35 \%$ & 0.010 \\
\hline WC & $0.194(0.068,0.321) *$ & $0.162(0.035,0.289) *$ & $0.030(0.014,0.050) *$ & $15.46 \%$ & 0.009 \\
\hline WHR & $0.162(0.054,0.269) *$ & $0.136(0.027,0.245) *$ & $0.031(0.015,0.053) *$ & $19.14 \%$ & 0.010 \\
\hline WHtR & $0.168(0.06 \mathrm{I}, 0.275) *$ & $0.139(0.030,0.248) *$ & $0.040(0.019,0.066) *$ & $23.81 \%$ & 0.012 \\
\hline \multicolumn{6}{|c|}{ Postmenopausal women } \\
\hline BMI & $0.155(-0.011,0.320)$ & $0.134(-0.036,0.305)$ & $0.028(0.011,0.049) *$ & Full mediating effect & 0.009 \\
\hline WC & $0.245(0.146,0.345) *$ & $0.224(0.123,0.325) *$ & $0.030(0.012,0.054) *$ & $12.24 \%$ & 0.011 \\
\hline WHR & $0.231(0.131,0.332) *$ & $0.211(0.109,0.312) *$ & $0.028(0.011,0.053) *$ & $12.12 \%$ & 0.010 \\
\hline WHtR & $0.232(0.131,0.333) *$ & $0.208(0.106,0.310) *$ & $0.035(0.014,0.064) *$ & $15.09 \%$ & 0.013 \\
\hline
\end{tabular}

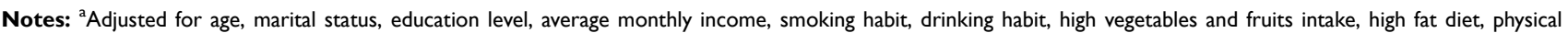
activity. *PE=indirect effect/total effect.

Abbreviations: hsCRP, high-sensitivity C-reactive protein; BMI, body mass index; WC, waist circumference; WHR, waist-to-hip ratio; WHtR, waist-to-height ratio; BFP, body fat percentage; VFI, visceral fat index.

obesity (defined by BMI), as well as diabetes mellitus, have been previously reported. ${ }^{35,36}$ Serum CRP has been associated with obesity, consistent with the results from other investigators' works. ${ }^{37,38}$ Recently, in a Mendelian randomization study, 5362 subjects were rolled to examine the potential causal relationship between CRP and obesity. Their results found that CRP was causally associated with BMI $[\beta$ $(95 \% \mathrm{CI})=0.98(0.32,1.63)], \mathrm{WC}[2.09(0.39,3.78)]$, and fat mass $[2.07(0.79,3.34)] .{ }^{39}$ Hotamisligil et al proposed that obesity was associated with low-grade inflammatory states, induced by different inflammatory factors. ${ }^{40} \mathrm{CRP}$ is widely implicated in the pathogenesis of metabolic disorders in obesity by regulating lipid metabolism and promoting fat synthesis. ${ }^{41,42}$ It also can affect the insulin-signaling pathway, stimulate insulin secretion, reduce tissue sensitivity to insulin, lead to systemic insulin resistance, and eventually promote obesity. ${ }^{43-47}$ Besides, CRP has been suggested as the main leptin-binding protein, and it could inhibit the function of leptin by interacting with the leptin receptor and lead to obesity. $^{35}$

Additionally, for the first time, this study provides evidence in humans that the association of progesterone and obesity is mediated by elevated serum hsCRP concentration. There are few studies on the association between progesterone and CRP. In one 8-year follow-up study from the Swedish, they studied the interrelation between steroid hormones and inflammation biomarkers in those oppositesex twins. ${ }^{48}$ The study, including 219 men and 183 women, found that progesterone was significantly associated with CRP (men: $r=0.267$; women: $r=0.150$ ). Several studies have found one ten-fold higher concentration of progesterone was related to a $19.4 \%$ or $23 \%$ increase of CRP in premenopausal women, ${ }^{22,49}$ and the use of progestin or medroxyprogesterone in the combination of estrogen among postmenopausal women significantly elevated the level of CRP. ${ }^{23,50}$ These findings can support our results to some extent. Progesterone has been reported to exhibit pro-inflammatory effects on neutrophils, ${ }^{51}$ through recruiting new neutrophils from the bone marrow and delaying apoptosis. ${ }^{52,53}$ However, the current results containing both men and postmenopausal women need further confirmation by more studies and the potential mechanism of progesterone-induced CRP needs to be more elucidated. Based on the mechanisms mentioned above, it seems reasonable that high progesterone levels lead to systemic inflammation, and in turn to obesity. ${ }^{39,40,54,55}$ And our mediation analysis testified our hypothesis and presented hsCRP significantly mediated the associations of progesterone and obesity.

Surprisingly we saw no significant association between progesterone with CRP and obesity in adult men aged $<46$ and $>65$ years old. It was reported that progesterone levels in men decline significantly with age. ${ }^{33}$ One epidemiologic study focuses aged 71-80 years individuals from Swedish find that the progesterone is not to be associated with metabolic diseases, which support our results. ${ }^{56}$ There 
was a negative correlation between progesterone and body weight $(r=-0.47)$ and BMI $(r=-0.58)$ in a sample of thirty-eight men aged 44.5 years old, ${ }^{19}$ which is inconsistent with our results. More studies will be needed to investigate this intriguing observation.

There are several strengths in the study. The major strength was the relatively large sample size including serum samples from 4687 participants (2474 men and 2213 postmenopausal women). Besides, mediation analysis was carried out to assess the underlying biological mechanisms linking progesterone to obesity. The significant mediation effect of systemic inflammatory marker on the linking provides an additional clue for pathogenesis. Finally, LC-MS/MS was used to measure progesterone concentration providing reliable results. However, several limitations should also be addressed. First, due to the cross-sectional study design, the causality between progesterone, hsCRP, and obesity could not be established. Second, we only examined progesterone levels, thus it potentially missed the possibility of other hormones and sex hormones levels. Third, the participants in the study came from rural areas of China, and these findings may not be generalizable to urban and other ethnic populations, based on possible differences in socioeconomic and genetic factors. Further studies with continued follow-up should be demanded. Despite these limitations, the current large population study still could reveal the important effects of CRP on progesterone and obesity.

\section{Conclusion}

This study observed that serum progesterone significantly increased the risk of obesity. The observed relationship between progesterone and obesity may have been mediated by hsCRP. This reflected systemic inflammation may be a potential mechanism underlying the association between progesterone and obesity. Further researches to confirm these findings and illustrate the potential mechanisms are urgently required.

\section{Abbreviations}

$\beta$, partial regression confident; BMI, body mass index; CI, confidence interval; CRP, C-reactive protein; hsCRP, highsensitivity C-reactive protein; IQR, inter-quartile range; LC-MS/MS, liquid chromatography-tandem mass spectrometry; Ln-, natural log-transformed; LOD, limit of detection; OR, odds ratio; Q1, the first quartile; Q2, the second quartile; Q3, the third quartile; Q4, the fourth quartile;
$\mathrm{RMB}$, renminbi; SD, standard deviation; WC, waist circumference; WHR, waist-to-hip ratio; WHtR, waist-toheight ratio.

\section{Data Sharing Statement}

The datasets generated during and/or analyzed during the current study are available from the corresponding author on reasonable request.

\section{Ethical Approval}

All procedures performed in studies involving human participants were in accordance with the ethical standards of the institutional and/or national research committee and with the 1964 Helsinki Declaration and its later amendments or comparable ethical standards. The study was approved by the Zhengzhou University Life Science Ethics Committee. (Code: [2015] MEC (S128)).

\section{Consent to Participate}

Informed consent was obtained from all individual participants included in the study.

\section{Consent for Publication}

The participant has consented to the submission.

\section{Acknowledgments}

We thank the study participants for their contributions to the study. We also express our gratitude for the research team for their contribution to the data collection and laboratory measurement.

\section{Author Contributions}

All authors made substantial contributions to conception and design, acquisition of data, or analysis and interpretation of data; took part in drafting the article or revising it critically for important intellectual content; agreed to submit to the current journal; gave final approval of the version to be published; and agree to be accountable for all aspects of the work.

\section{Funding}

This research was supported by the National Natural Science Foundation of China (Grant NO: 21806146), the National Key Research and Development Program of China (Grant NO: 2016YFC0900803, 2019YFC1710 002), the Henan Science and Technology Development Program (Grant No: 182102310562, 202102310046), the 
Postdoctoral Science Foundation of China (Grant NO: 2016M602264, 2020T130604), and the Excellent Youth Development Foundation of Zhengzhou University (Grant No: 2018ZDGGJS052).

\section{Disclosure}

The authors report no conflicts of interest in this work.

\section{References}

1. Guglielmi V, Sbraccia P. Obesity phenotypes: depot-differences in adipose tissue and their clinical implications. Eat Weight Disord. 2018;23:3-14. doi:10.1007/s40519-017-0467-9

2. Wang YC, McPherson K, Marsh T, Gortmaker SL, Brown M. Health and economic burden of the projected obesity trends in the USA and the UK. Lancet. 2011;378:815-825. doi:10.1016/S0140-6736(11) 60814-3

3. Dwivedi AK, Dubey P, Cistola DP, Reddy SY. Association between obesity and cardiovascular outcomes: updated evidence from meta-analysis studies. Curr Cardiol Rep. 2020;22:25. doi:10.1007/ s11886-020-1273-y

4. World Health Organization. http://www.who.int/mediacentre/fact sheets/fs311/en/.

5. Liu X, Wu W, Mao Z, et al. Prevalence and influencing factors of overweight and obesity in a Chinese rural population: the Henan Rural Cohort Study. Sci Rep. 2018;8:13101. doi:10.1038/s41598018-31336-2

6. Keaver L, Webber L, Dee A, Shiely F, Marsh T, Balanda K. Application of the UK foresight obesity model in Ireland: the health and economic consequences of projected obesity trends in Ireland. PLoS One. 2013;8:e79827. doi:10.1371/journal.pone.0079827

7. Federation ID: The IDF consensus worldwide definition of the metabolic syndrome. 2006.

8. Srinivasan SR, Wang R, Chen W, Wei CY, Xu J, Berenson GS. Utility of waist-to-height ratio in detecting central obesity and related adverse cardiovascular risk profile among normal weight younger adults (from the Bogalusa Heart Study). Am J Cardiol. 2009; 104:721-724. doi:10.1016/j.amjcard.2009.04.037

9. Chen C, Lu FC. Department of Disease Control Ministry of Health PRC: the guidelines for prevention and control of overweight and obesity in Chinese adults. Biomed Environ Sci. 2004;17:Suppl:1-36.

10. Organization WH. Waist Circumference and Waist-Hip Ratio Report of a WHO Expert Consultation. Geneva; 2008.

11. Fu W, Cao S, Liu B, et al. Association of general and central adiposity with blood pressure among Chinese adults: results from the China National Stroke Prevention Project. J Hypertens. 2018;36:2406-2413. doi:10.1097/HJH.0000000000001852

12. Bragg F, Tang K, Guo Y, et al. Associations of general and central adiposity with incident diabetes in Chinese men and women. Diabetes Care. 2018;41:494-502. doi:10.2337/dc17-1852

13. Dale CE, Fatemifar G, Palmer TM, et al. Causal associations of adiposity and body fat distribution with coronary heart disease, stroke subtypes, and Type 2 diabetes mellitus: a mendelian randomization analysis. Circulation. 2017;135(24):2373-2388. doi:10.1161/CIRCULATIONAHA.116.026560

14. Campbell SE, Febbraio MA. Effects of ovarian hormones on exercise metabolism. Curr Opin Clin Nutr Metab Care. 2001;4:515-520. doi:10.1097/00075197-200111000-00009

15. Mayes JS, Watson GH. Direct effects of sex steroid hormones on adipose tissues and obesity. Obes Rev. 2004;5(4):197-216. doi:10.11 11/j.1467-789X.2004.00152.x
16. Gray JM, George WN. Cytoplasmic progestin binding in rat adipose tissues. Endocrinology. 1979;104(5):1377-1382. doi:10.1210/endo104-5-1377

17. Esfahlan RJ, Zarghami N, Esfahlan AJ, Mollazadeh M, Nejati K, Nasiri M. The possible impact of obesity on androgen, progesterone and estrogen receptors (ERalpha and ERbeta) gene expression in breast cancer patients. Breast Cancer (Auckl). 2011;5:227-237. doi:10.4137/BCBCR.S7707

18. Clark MK, Dillon JS, Sowers M, Nichols S. Weight, fat mass, and central distribution of fat increase when women use depot-medroxyprogesterone acetate for contraception. Int $J$ Obes (Lond). 2005;29:1252-1258. doi:10.1038/sj.ijo.0803023

19. Blanchette S, Marceau P, Biron S, Brochu G, Tchernof A. Circulating progesterone and obesity in men. Horm Metab Res. 2006;38: 330-335. doi:10.1055/s-2006-925392

20. Goh JY, He S, Allen JC, Malhotra R, Tan TC. Maternal obesity is associated with a low serum progesterone level in early pregnancy. Horm Mol Biol Clin Investig. 2016;27:97-100. doi:10.1515/hmbci2015-0030

21. Moutachakkir M, Lamrani Hanchi A, Baraou A, Boukhira A, Chellak S. Immunoanalytical characteristics of C-reactive protein and high sensitivity C-reactive protein. Ann Biol Clin (Paris). 2017;75:225-229. doi:10.1684/abc.2017.1232

22. Gaskins AJ, Wilchesky M, Mumford SL, et al. Endogenous reproductive hormones and C-reactive protein across the menstrual cycle: the BioCycle Study. Am J Epidemiol. 2012;175:423-431. doi:10.10 93/aje/kwr343

23. Reuben DB, Palla SL, Hu P, et al. Progestins affect mechanism of estrogen-induced C-reactive protein stimulation. $A m \quad J$ Med. 2006;119:167 e161-168. doi:10.1016/j.amjmed.2005.08.012

24. Black S, Kushner I, Samols D. C-reactive Protein. J Biol Chem. 2004;279:48487-48490. doi:10.1074/jbc.R400025200

25. Berg AH, Scherer PE. Adipose tissue, inflammation, and cardiovascular disease. Circ Res. 2005;96:939-949. doi:10.1161/01.RES.0000 163635.62927.34

26. Fearnley GR, Chakrabarti R, Avis PRD. Blood fibrinolytic activity in diabetes mellitus and its bearing on ischaemic heart disease and obesity. Br Med J. 1963;1:921-923. doi:10.1136/bmj.1.5335.921

27. Liu X, Mao Z, Li Y, et al. Cohort Profile: the Henan Rural Cohort: a prospective study of chronic non-communicable diseases. Int J Epidemiol. 2019;48:1756-1756j. doi:10.1093/ije/dyz039

28. Pearson TA, Mensah GA, Alexander RW, et al. Markers of inflammation and cardiovascular disease: application to clinical and public health practice: a statement for healthcare professionals from the Centers for Disease Control and Prevention and the American Heart Association. Circulation. 2003;107:499-511. doi:10.1161/01.CIR.00 00052939.59093 .45

29. Society CN. The Dietary Guidelines for Chinese Residents. The Tibet People's Publishing House. Lhasa; 2011.

30. China WGoOi. Guidelines for prevention and control of overweight and obesity in chinese adults. Acta Nutrimenta Sinica. 2004;26.

31. Glisic M, Shahzad S, Tsoli S, et al. Association between progestin-only contraceptive use and cardiometabolic outcomes: a systematic review and meta-analysis. Eur J Prev Cardiol. 2018;25:1042-1052. doi:10.1177/2047487318774847

32. Vanderweele TJ, Vansteelandt S. Odds ratios for mediation analysis for a dichotomous outcome. Am J Epidemiol. 2010;172:1339-1348. doi:10.1093/aje/kwq332

33. Genazzani AR, Petraglia F, Bernardi F, et al. Circulating levels of allopregnanolone in humans: gender, age, and endocrine influences. J Clin Endocrinol Metab. 1998;83:2099-2103. doi:10.1210/jcem.83. 6.4905

34. Wu Y, Potempa LA, El Kebir D, Filep JG. C-reactive protein and inflammation: conformational changes affect function. Biol Chem. 2015;396:1181-1197. 
35. Sudhakar M, Silambanan S, Chandran AS, Prabhakaran AA, Ramakrishnan R. C-Reactive Protein (CRP) and leptin receptor in obesity: binding of monomeric crp to leptin receptor. Front Immunol. 2018;9:1167. doi:10.3389/fimmu.2018.01167

36. Freeman DJ, Norrie J, Caslake MJ, et al. West of Scotland Coronary Prevention S: c-reactive protein is an independent predictor of risk for the development of diabetes in the West of Scotland Coronary Prevention Study. Diabetes. 2002;51:1596-1600. doi:10.2337/diabetes. 51.5.1596

37. Meisel P, Eremenko M, Holtfreter B, Volzke H, Kocher T. The sex paradox in the interplay between periodontitis, obesity, and serum C-reactive protein: data from a general population. $J$ Periodontol. 2019;90:1365-1373. doi:10.1002/JPER.18-0733

38. Ebrahimi M, Heidari-Bakavoli AR, Shoeibi S, et al. Association of Serum hs-CRP levels with the presence of obesity, diabetes mellitus, and other cardiovascular risk factors. J Clin Lab Anal. 2016;30 (5):672-676. doi:10.1002/jcla.21920

39. Bochud M, Marquant F, Marques-Vidal PM, et al. Association between C-reactive protein and adiposity in women. $J$ Clin Endocrinol Metab. 2009;94:3969-3977. doi:10.1210/jc.2008-2428

40. Hotamisligil GS. Inflammatory pathways and insulin action. Int J Obes Relat Metab Disord. 2003;27(Suppl 3):S53-55. doi:10. 1038/sj.ijo.0802502

41. Nieto-Vazquez I, Fernandez-Veledo S, Kramer DK, Vila-Bedmar R, Garcia-Guerra L, Lorenzo M. Insulin resistance associated to obesity: the link TNF-alpha. Arch Physiol Biochem. 2008;114(3):183-194. doi:10.1080/13813450802181047

42. Kovacs A, Green F, Hansson LO, et al. A novel common single nucleotide polymorphism in the promoter region of the C-reactive protein gene associated with the plasma concentration of C-reactive protein. Atherosclerosis. 2005;178:193-198. doi:10.1016/ j.atherosclerosis.2004.08.018

43. Kim KA. Recent progress in research on beta-cell apoptosis by cytokines. Front Biosci (Landmark Ed). 2009;14:657-664. doi:10. 2741/3271

44. Novotny GW, Lundh M, Backe MB, et al. Transcriptional and translational regulation of cytokine signaling in inflammatory beta-cell dysfunction and apoptosis. Arch Biochem Biophys. 2012;528: 171-184. doi:10.1016/j.abb.2012.09.014

45. Ndumele CE, Pradhan AD, Ridker PM. Interrelationships between inflammation, C-reactive protein, and insulin resistance. J Cardiometab Syndr. 2006;1:190-196. doi:10.1111/j.1559-4564.20 06.05538.x
46. Pradhan AD, Manson JE, Rifai N, Buring JE, Ridker PM. C-reactive protein, interleukin 6 , and risk of developing type 2 diabetes mellitus. JAMA. 2001;286:327-334. doi:10.1001/jama.286.3.327

47. Barazzoni R, Gortan Cappellari G, Ragni M, Nisoli E. Insulin resistance in obesity: an overview of fundamental alterations. Eat Weight Disord. 2018;23:149-157. doi:10.1007/s40519-018-0481-6

48. Brismar K, Nilsson SE. Interrelations and associations of serum levels of steroids and pituitary hormones with markers of insulin resistance, inflammatory activity, and renal function in men and women aged $>70$ years in an 8-year longitudinal study of oppositesex twins.. Gend Med. 2009;6(Suppl 1):123-136. doi:10.1016/j. genm.2009.01.001

49. Wander K, Brindle E, O’Connor KA. C-reactive protein across the menstrual cycle. Am J Phys Anthropol. 2008;136:138-146. doi:10. 1002/ajpa.20785

50. Walsh BW, Paul S, Wild RA, Dean RA, Tracy RP, Cox DA. The effects of hormone replacement therapy and raloxifene on $\mathrm{C}$-reactive protein and homocysteine in healthy postmenopausal women: a randomized, controlled trial. J Clin Endocrinol Metab. 2000; 85:214-218. doi:10.1210/jcem.85.1.6326

51. Bouman A, Heineman MJ, Faas MM. Sex hormones and the immune response in humans. Hum Reprod Update. 2005;11:411-423. doi:10. 1093/humupd/dmi008

52. Bain BJ, England JM. Variations in leucocyte count during menstrual cycle. $\mathrm{Br}$ Med J. 1975;2:473-475. doi:10.1136/bmj.2.5969.473

53. Molloy EJ, O'Neill AJ, Grantham JJ, Sheridan-Pereira M, Fitzpatrick JM, Webb DW. Sex-specific alterations in neutrophil apoptosis: the role of estradiol and progesterone. Blood. 2003;10 2:2653-2659. doi:10.1182/blood-2003-02-0649

54. Manson JE, Hsia J, Johnson KC, et al. Estrogen plus progestin and the risk of coronary heart disease. $N$ Engl J Med. 2003;349:523-534. doi:10.1056/NEJMoa030808

55. Ishii S, Karlamangla AS, Bote M, Irwin MR, Jacobs DRJr, Cho HJ. Gender, obesity and repeated elevation of C-reactive protein: data from the CARDIA cohort. PLoS One. 2012;7:e36062. doi:10.1371/ journal.pone.0036062

56. Nilsson SE, Fransson E, Brismar K. Relationship between serum progesterone concentrations and cardiovascular disease, diabetes, and mortality in elderly Swedish men and women: an 8-year prospective study. Gend Med. 2009;6:433-443. doi:10.1016/j.genm.20 09.09.011
Journal of Inflammation Research

\section{Publish your work in this journal}

The Journal of Inflammation Research is an international, peerreviewed open-access journal that welcomes laboratory and clinical findings on the molecular basis, cell biology and pharmacology of inflammation including original research, reviews, symposium reports, hypothesis formation and commentaries on: acute/chronic inflammation; mediators of inflammation; cellular processes; molecular mechanisms; pharmacology and novel anti-inflammatory drugs; clinical conditions involving inflammation. The manuscript management system is completely online and includes a very quick and fair peerreview system. Visit http://www.dovepress.com/testimonials.php to read real quotes from published authors. 